\title{
Particle Swarm Optimization Algorithms for Two-stage Hybrid Flowshop Scheduling Problem with No-wait
}

\author{
Mageed A. Ghaleb, Ibrahim M. Alharkan* \\ Industrial Engineering Department, King Saud University, Saudi Arabia
}

Copyright $(2019$ by authors, all rights reserved. Authors agree that this article remains permanently open access under the terms of the Creative Commons Attribution License 4.0 International License

\begin{abstract}
Hybrid flowshop scheduling problems have attracted much attention owing to their wide applications in a variety of real-world problems. In some industries, products cannot be allowed to wait between any consecutive productions stages, which illustrates the importance of studying the no-wait constraint. One of the reasons for such a constraint is that, for some products, the waiting time could cause permanent damage. However, the no-wait constraint was neglected in many prior studies, which in some production environments may not be allowed. Minimizing the total tardiness plays a key role in making scheduling decisions to meet customers' due dates. In this study, we solve the no-wait two-stage hybrid flowshop scheduling problem with total tardiness minimization as an optimizing criterion. We formulated the problem mathematically and proposed two discrete versions of the particle swarm optimization (PSO) to solve it. Moreover, three discrete versions of PSO are adopted from previous studies and used as benchmarks to test the effectiveness of the two proposed algorithms. Compared to the benchmark algorithms, the results showed that the two newly proposed algorithms were effective and performed better than the benchmark algorithms in terms of the average relative error. The current study represents one of the few attempts to investigate the considered problem with total tardiness minimization, as well as introducing new and effective discrete versions of the PSO algorithms to solve the problem under investigation.
\end{abstract}

Keywords Scheduling, Hybrid Flowshop, No-wait, Total Tardiness, Particle Swarm Optimization

\section{Introduction}

Consider a two-stage hybrid flowshop with a set of $n$ jobs (or operations) needed to be scheduled, taking in account that in each stage there is more than one machine. The machines are identical and in parallel. At each stage, each job is performed on only one of the existing machines. Each job has a completion time on stage one that is equal to its start time on stage two. In other words, jobs are not allowed to wait between the two stages and have to be moved from stage one to stage two without any interruptions. This problem is known as the two-stage nowait hybrid flowshop scheduling problem (NWHFSP).

The two-stage NWHFSP has several industrial in industry. Therefore, it has received much attention from many researchers. For instance, [1] presented an application to the synthetic fiber industry. [2] described an application to a computer system that has a single server and two parallel processors. [3] described an application to an annealing roller in a case steel plant. [4] addressed an application to semiconductor packaging. Generally, in any manufacturing firm with a production line that consists of consecutive production stages with parallel identical machines in at least one of those stages, we can consider the allocation of items to be produced in that line with a nowait restriction between its consecutive stages as a NWHFSP. Hence, the two-stage NWHFSP is realistic and occurs in many real-life applications.

The simplest hybrid system, which is a two-stage hybrid flowshop with only one machine in one of its stages and several identical parallel machines in the other stage, has been addressed in many previous studies. For instance, [5] developed a greedy algorithm, named least deviation algorithm (LDA), to minimize the makespan in the simplest two-stage NWHFSP. [6] developed two heuristic algorithms to minimize the makespan in the simplest twostage NWHFSP with separate setup and removal times. [7] designed a genetic algorithm (GA) to minimize the makespan in the simplest two-stage NWHFSP. [8] developed a branch and bound (B\&B) algorithm to minimize the makespan in the simplest two-stage NWHFSP.

Problems with setup times, random machines breakdowns, and rework probability have also been investigated in previous studies. For example, [4] formulated the problem as an integer programming model and adopted an ant colony optimization (ACO) algorithm to minimize the total completion time in a two-stage NWHFSP with setup times. [9] Utilized a particle swarm 
optimization (PSO) and imperialist competitive algorithm (ICA) to minimize the maximum tardiness in a two-stage NWHFSP with setup times and rework probability. [10] introduced a GA and an adaptive ICA (AICA) to minimize the total completion time in a two-stage NWHFSP with setup times. [11] designed a hybrid algorithm based on ICA to minimize the makespan in a two-stage NWHFSP with unrelated parallel machines, sequence-dependent setup times, rework probability, and ready times. [12] proposed a GA and simulated annealing (SA) algorithms to minimize the makespan in a two-stage NWHFSP with group sequence-dependent setup times and random machines breakdowns.

The precedence constraint and the bi-objective two-stage NWHFSP have also been addressed in previous investigations. For example, [13] used an adaptive randomized list scheduling heuristic (ARLS) to minimize the makespan in a two-stage NWHFSP with precedence constraints. [14] addressed three SA-based algorithms to solve a bi-objective two-stage NWHFSP, where the considered objectives were the makespan and maximum tardiness.

The general two-stage hybrid system with several identical parallel machines in either stage has also been investigated in many studies. For instance, [1] developed a branch and bound (B\&B) algorithm to minimize the makespan in a two-stage NWHFSP. Then, [15] presented several heuristic algorithms to solve a two-stage NWHFSP with makespan minimization, where these algorithms were analyzed in the context of the worst-case performance. [16] addressed a heuristic algorithm named the minimum deviation algorithm (MDA) to minimize the makespan in a two-stage NWHFSP. [2] proposed an exact algorithm to minimize the total completion time in a two-stage NWHFSP; the proposed algorithm obtained the optimal solution in $O(n \log (n))$ time. [17] developed a heuristic algorithm named MLTP to minimize the makespan in a two-stage NWHFSP with no idle machines. [3] formulated the problem of minimizing the makespan in a two-stage NWHFSP as an integer-programming model and proposed a discrete PSO (DPSO) to solve it. [18] utilized the SA and GA to solve the two-stage NWHFSP with makespan minimization. [19] used six heuristic algorithms to minimize the makespan in a two-stage NWHFSP, where an adaptive neuro-fuzzy interface system (ANFIS) was applied for makespan estimation. [20] developed six metaheuristic algorithms based on ICA, ACO, and PSO to minimize mean flow time in a two-stage NWHFSP. [21] proposed a tabu search (TS) and several discrete versions of PSO algorithms to minimize the makespan in a twostage NWHFSP.

Based on the literature review, we found that research studies have solved the two-stage NWHFSP with different performance measures. A large portion of these measures was related to the completion time, such as the mean flow time, total completion time, and makespan. On the contrary, a small portion of these measures was related to the due date, such as the lateness, tardiness, and number of tardy jobs. Moreover, the due date-related performance measure considered was the maximum tardiness. Despite the significance of minimizing the total tardiness in many environments, especially when penalties are applied for jobs delivered beyond their due dates [22], few previous studies have aimed to minimize the total tardiness in the two-stage NWHFSP. Therefore, in this study, we considered the minimization of the total tardiness as a performance measure. It was also found that few studies considered the use of the PSO to solve the two-stage NWHFSP. Moreover, the proposed PSO algorithms were simple adoptions of well-known discrete versions of the PSO, with no significant improvements. Hence, in this study, we introduced some problem-related improvements to the PSO algorithms found in the literature and adopted them to solve our problem. In addition, we introduced two new discrete versions of the PSO algorithm for the problem under investigation. The three adopted algorithms are used as benchmarks to evaluate the performance of the two newly introduced algorithms. The problem is formulated mathematically as a mixed integer programming model, based on the model addressed by [14].

The following section describes the problem, presents a mathematical model formulation, and discusses the complexity of the problem. Five algorithms are explained in Section 3 and a comparison study is presented in Section 4. Finally, we draw our conclusions and address directions for future research in Section 5 .

\section{Problem Formulation}

For each job in the set of $n$ available jobs, two operations are required to finish processing. The first is conducted on one of the $m_{1}$ identical parallel machines available at stage one, but under the condition that there will be an available machine at stage two to process that job as soon as it finishes its processing in the first stage (the nowait restriction). The second operation is completed by one of the $m_{2}$ identical parallel machines available at stage two with zero waiting time between the two stages. The objective is to minimize the total tardiness. The problem can be represented as in Figure 1, and schematically using Graham's notation $(\alpha|\beta| \gamma)$ as $F\left(m_{1}, m_{2}\right)|n w t| \sum T_{j}$, where the term $F\left(m_{1}, m_{2}\right)$ stands for two-stage hybrid flowshop with $m_{1}$ identical parallel machines in the first stage and $m_{2}$ identical parallel machines in the second. The symbol $n w t$ stands for the no-wait restriction. $\sum T_{j}$ is the total tardiness, which is the measurement criterion. 


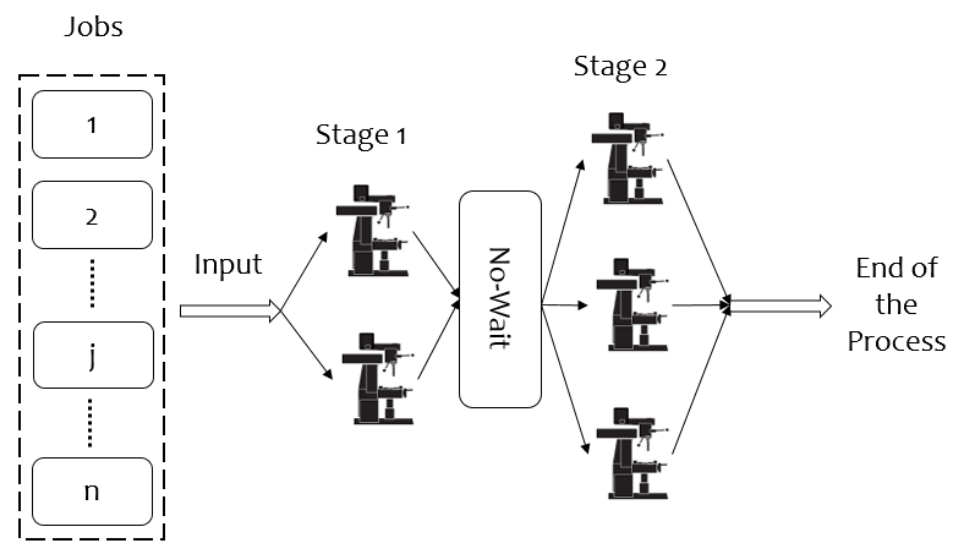

Figure 1. Two-stage Hybrid Flowshop Environment with No-wait (nwt) Restriction.

The following assumptions are made:

- $\quad$ All data in all problems used are assumed to be integer and deterministic.

- $\quad$ Each stage has more than one machine (machines are identical and in parallel).

- $\quad$ All jobs are available at time zero, and preemptions are not allowed.

- $\quad$ Processing times required at each stage are known in advance.

- $\quad$ Set-up times are all included in the processing times.

- Transportation times between the stages are negligible.

- Machines are continuously available.

- Waiting time between two consecutive stages for each job is zero (no-wait).

A mixed integer programming model based on the model presented by [14] is given with the following variables:

- $n$ Number of jobs to be scheduled

- $\quad S$ Number of stages, $S=2$

- $m_{i}$ Number of identical parallel machines at stage $i$, where $i=1, \ldots, S$

- $\quad p_{i, j}$ Processing time of job $j$ at stage $i$, where $j=$ $1, \ldots, n$

- $d_{j}$ Due date of job $j$

- $\quad t_{i, j}^{k}$ Available time for job $j$ at stage $i$ on machine $k$, where $k=1, \ldots, m_{i}$

- $\quad w_{1 j}^{k}$ Delay time for job $j$ on machine $k$ at stage 1 (to satisfy the no-wait constraint)

- $x_{j l} \quad 1$ if job $j$ is located in position $l$ of the job sequence

- $\quad y_{i j k} 1$ if job $j$ is processed on machine $k$ at stage $i ; 0$ otherwise

- $s_{i j} \quad$ Starting time of job $j$ at stage $i$

- $\quad c_{i j}$ Completion time of job $j$ at stage $i$

- $T_{j}$ Tardiness of job $j$, equal $\max \left(0, c_{2 j}-d_{j}\right)$

Objective function:

$$
\min z=\sum_{j=1}^{n} T_{j}
$$

Subject to:

$$
\begin{gathered}
\sum_{j=1}^{n} x_{j l}=1 \quad l=1,2, \ldots, n \\
\sum_{l=1}^{n} x_{j l}=1 \quad j=1,2, \ldots, n \\
\sum_{k=1}^{m_{i}} y_{i j k}=1 \quad i=1,2 \text { and } j=1,2, \ldots, n \\
w_{1 j}^{k}=\max \left(0, t_{2, j}^{k}-t_{1, j}^{k}\right) \quad j=1,2, \ldots, n \\
s_{1, j}=t_{1, j}^{k}+w_{1 j}^{k} \quad j=1,2, \ldots, n \\
c_{1, j}=s_{2, j}=s_{1, j}+p_{1, j} \quad j=1,2, \ldots n \\
c_{2, j}=s_{2, j}+p_{2, j} \quad j=1,2, \ldots, n \\
\sum_{j=1}^{n} x_{j l} s_{i j} \leq \sum_{j}^{n} x_{j(l+1)} s_{i j} i=1,2 \text { and } l=1,2, \ldots, n \\
\left(c_{2 j}-d_{j}\right)^{+} \leq T_{j} \quad j=1,2, \ldots, n, \text { and }(X)^{+} \text {is } \max (0, X)
\end{gathered}
$$

Constraints (2) and (3) ensure that every schedule is a permutation of all jobs. The fact that each job can be operated on only one machine in any stage is captured in constraint (4). Constraint (5) calculates the delays that may occur as a result of the no-wait restriction. Constraint (6) indicates the starting time for each job to satisfy the no-wait constraint. The job completion times in each stage are computed using constraints (7) and (8). Constraint (7) also ensures that the starting time of each job in stage 2 is equal to its completion time at stage 1 (the no-wait constraint). Constraint (9) enforces the precedence constraints in the sequence. The tardiness values are captured via constraint (10).

In general, hybrid flowshops are difficult to solve because of the resulting combinatorial explosion with the increase in problem size. According to [23], for an $S \times$ $n$ (stages $\times$ jobs $)$ HFSP with $m_{i}$ parallel machines at stage $i$, the total number of possible solutions is $n !\left(\prod_{i=1}^{S} m_{i}\right)^{n}$. In particular, [24] proved that $F(1, m) \| C_{\text {max }}$ is NP-hard in the strong sense for $m \geq 2$. $F(1, m) \| C_{\max }$ is a special case of $\left.F\left(m_{1}, m_{2}\right)|n w t| \sum T_{j}\right)$; therefore

LEMMA $1 F\left(m_{1}, m_{2}\right)|n w t| \sum T_{j}$ is NP-hard in the strong sense.

The complexity of the concerned problem makes it 
worthwhile to investigate the use of metaheuristics to solve it, which is the aim of this study.

\section{Solution Approaches}

The PSO algorithm is one of the recently developed metaheuristics. It was first introduced by [25] to optimize various continuous nonlinear functions. It was designed based on the metaphor of social interaction and communication such as in bird flocks and fish schools. Recently, PSO has been used successfully in a wide range of applications such as the traveling salesman problem [26], vehicle routing [27], and scheduling [28].

PSO has several advantages over other known metaheuristics, which include structural simplicity, implementation ease, and rapid speed of acquiring solutions [29]. Despite its advantages, the applications of PSO to the NWHFSP are limited compared to other algorithms such as GA and SA. However, the continuous nature of the PSO algorithm is the major obstacle of its successful application to this problem. To remedy this drawback, several researchers have suggested different ways to encode a schedule (or generally a permutation) to a PSO particle, which lead to different PSO variants to solve the problem.

There are no previous reports of using PSO for minimizing the total tardiness in the two-stage NWHFSP. However, for other optimization criteria, [9] proposed a PSO algorithm for the problem with maximum tardiness minimization, [3] addressed a PSO algorithm for the problem with makespan minimization, and [20] presented a PSO algorithm for the problem with mean flow time minimization. Moreover, in previous research, [21] proposed several discrete versions of PSO algorithms, based on the three abovementioned algorithms, to minimize the makespan. The authors reported that the performance of the addressed PSO algorithms was promising compared to other designed algorithms, which motivates us to adopt these algorithms and use them as benchmarks to evaluate the newly introduced algorithms in Section 3.2. Three different variants of the PSO were adopted and denoted as ST-PSO [3], [21], SMR-PSO [20], [21], and MSRM-PSO [9], [21]. Details of the three adopted algorithms (ST-PSO, SMR-PSO, and MSRM-PSO) are presented in Section 3.1.

\subsection{ST-PSO, SMR-PSO, and MSRM-PSO Algorithms}

[3] proposed a discrete PSO algorithm to minimize the makespan in the two-stage NWHFSP. The proposed algorithm is a standard PSO with a sequential coding method that converts particles into feasible permutations. The method simply takes an updated particle (after the particle's velocity and position are updated in the standard PSO) and sequentially replaces the unfeasible elements of the particle with feasible elements, such that the resulted particle is converted into a permutation. We adapted the developed algorithm to our problem and we denote it as STPSO. [20] also proposed a discrete PSO algorithm for the problem with mean flow time minimization. The proposed algorithm is also a standard PSO with a value-ranked rule that converts particles into feasible permutations. The coding scheme is similar to that in [3], except that the rule used here is a value-ranked rule that gives priority to particle elements based on their values. The element with the lowest value has the highest priority, and then a permutation is developed based on elements' priorities. The algorithm was adapted to our problem and denoted as SMR-PSO. Another PSO algorithm was also proposed by [9] to solve the problem with maximum tardiness minimization. The proposed PSO was also a standard PSO algorithm with a particle update scheme that is generally inspired by the mutation and crossover operators of evolutionary algorithms. The particle update process consists of three steps; the first and second steps were conducted by applying a crossover operator, and the third step was to apply a mutation operator to the resulting particle from the previous two steps. The algorithm was also adapted and we denoted it as MSRM-PSO. As mentioned earlier, these three algorithms were adopted in the previous work of [21] and compared to a TS algorithm.

The following improvements were introduced to the three adopted algorithms (ST-PSO, SMR-PSO, and MSRM-PSO), and the details are presented in Section 3.2:

- Two problem-specific initial particles: the first is based on an earliest due date (EDD) rule and the second is based on a modified MDA.

- The final particle update is a combination of the insertion and swap mutation operators.

For the sake of brevity, the details of the three adopted algorithms are not given here, but the reader can refer to the original papers for more information about the employed coding schemes to encode the PSO particles into permutations. The stopping criterion in all the adopted and developed algorithms was the computation time (in seconds), which was set to be equal for all algorithms. Moreover, for any instance, if zero total tardiness is found (even if before reaching the time specified as the main stopping criterion), the algorithm stops and the computational time (in seconds) is reported.

\subsection{Proposed Algorithms: IGPSO and HGPSO}

The geometric particle swarm optimization (GPSO) is an important discrete version of the PSO algorithm that was first introduced by [30] for solving constrained combinatorial problems. GPSO bridges the gap between PSO and evolutionary algorithms. A new crossover operator was introduced that simply generalizes PSO to virtually any combinatorial solution representation. The new GPSO is applicable to both continuous and combinatorial spaces. 
A particle $i$ at iteration $t$ is updated in the standard PSO in two steps. The first is to update particle's velocity $v_{i}(t)$ through its previous velocity $v_{i}(t-1)$, the distance between its previous position $x_{i}(t-1)$ and its best position $p_{i}$, and the distance between its previous position $x_{i}(t-1)$ and the global best position $p_{g}$. Then, the particle's position $x_{i}$ is updated using its previous position $x_{i}(t-1)$ and the new velocity $v_{i}(t)$. Three parents (as inspired from evolutionary algorithms) are incorporated in the update process of any particle. The key issue in the GPSO is the direct update of the particle's position instead of using the velocity concept. The newly developed crossover operator in the GPSO enables the possibility of producing new offspring by crossing more than two parents at the same time, not only two parents as with regularly known crossover operators in evolutionary algorithms. In the present case, the particle update in the standard PSO requires crossing three parents, which are the particle's current position $x_{i}$, particle's best position $p_{i}$, and global best position $p_{g}$. Therefore, a three-parent mask-passed crossover operator (3PMX) was applied to each particle to move it to its new position using the flowing equation:

$x_{i}(t)=3 P M X\left(\left(x_{i}(t-1), w_{1}\right),\left(p_{i}(t-\right.\right.$

$\left.\left.1), w_{2}\right),\left(p_{g}, w_{3}\right)\right)$

Here,

The particle $x_{i}(t)$ corresponds to a permutation $\pi_{i}(t)$.

The factor $t$ represents the iterative generation number.

$3 P M X$ is the newly developed crossover by [30].

$w_{1}, w_{2}$, and $w_{3}$ are weight values that indicate for each element in the mask, the probability of having values from the parents' $x_{i}, p_{i}$, or $p_{g}$, respectively.

Then, the particle's new position was updated using a mutation operator to produce a new particle at the neighborhood of the resulted particle's position from Eq. 13. The following example illustrates the new particle update process in GPSO using 3PMX.

\section{Example 1:}

Consider having the following weights $w_{1}=0.2, w_{2}=$ 0.4 , and $w_{3}=0.4$ for parents $x_{i}, p_{i}$ and $p_{g}$ respectively. Moreover, the sequences related to the three parents at iteration $(t-1)$ are $x_{i}=(1,3,4,5,2), p_{i}=(3,4,2,1,5)$, and $p_{g}=(2,5,3,4,1)$. Find the new particle position $x_{i}(t)$ using 3 PMX.

\section{Solution:}

The first step is to randomly generate the mask as a vector of the same length as the parents, which is five elements in this example. The mask elements are one of three values $(1,2$, or 3$)$ because we have only three parents. The elements in the mask are proportional to the recombination weights $w_{1}, w_{2}$, and $w_{3}$ of the parents. The generated mask based on the given data is Mask= $(2,1,3,3,2)$, which represents $20 \%$ of parent $1,40 \%$ of parent 2 , and $40 \%$ of parent 3 .

\begin{tabular}{cc}
\hline Mask & 21332 \\
$x_{i}(t-1)$ & 13452 \\
$p_{i}(t-1)$ & 34215 \\
$p_{g}$ & 25341 \\
$x_{i}(t)$ & $x \times x \times$ \\
\hline
\end{tabular}

For any parent, the content of a position is changed, based on the mask value at that position, by swapping it with the content of another position in the parent. By scanning the mask from right to left, the swapping process can be illustrated as follows: In position 1, the mask has a value of "2." This means that position 1 in parents $x_{i}(t-$ 1) and $p_{g}$ must become equal to the value of position 1 in parent $p_{i}(t-1)$. This is done by swapping elements 1 and 2 in parent $x_{i}(t-1)$ and elements 1 and 3 in parent $p_{g}$.

\begin{tabular}{cc}
\hline Mask & 21332 \\
$x_{i}(t-1)$ & 31452 \\
$p_{i}(t-1)$ & 34215 \\
$p_{g}$ & 35241 \\
$x_{i}(t)$ & $3 \times x \times x$ \\
\hline
\end{tabular}

The recombination now continues the updated parents: parent $p_{i}(t-1)$ is left unchanged and the current parent $x_{i}(t-1)$ and parent $p_{g}$ are the original parents' $x_{i}(t-$ 1) and $p_{g}$ after the swap. Hence, the elements are fixed after considering all the positions (when the mask has been completely scanned). The resulting permutation is the offspring permutation. Moving from position 2 to 5 using the same method conducted at position 1 results in the following offspring.

\begin{tabular}{cl}
\hline Mask & 21332 \\
$x_{i}(t-1)$ & 31245 \\
$p_{i}(t-1)$ & 31245 \\
$p_{g}$ & 31245 \\
$x_{i}(t)$ & 31245 \\
\hline
\end{tabular}

We notice that the resulted offspring is generated by a recombination process that uses multiple sorting of the three parents toward each other according to the contents of the crossover mask. This recombination can be easily generalized to any number of parents.

Using 3PMX as illustrated in the previous example, we proposed two variants of PSO. The first is a GPSO algorithm with the following improvements:

- Two problem-related initial particles: the first is based on an earliest due date (EDD) rule and the second is based on a modified MDA.

- The final particle update is a combination of the insertion and swap mutation operators.

The first algorithm is denoted as improved GPSO (IGPSO), and the second is a hybrid GPSO algorithm that uses a simulated annealing (SA) algorithm to optimize its initial swarms before the updating process. The second 
algorithm is denoted as HGPSO. We start by describing the modified MDA and the IGPSO algorithm before explaining the HGPSO algorithm.

The MDA was originally developed by [16] to solve the $F\left(m_{1}, m_{2}\right)|n w t| C_{\max }$. We simply modify the algorithm by adopting the EDD rule to break ties if more than one job is found to be ready for processing, such that ties are now broken using the EDD rule instead of being broken arbitrarily. Moreover, we calculate the total tardiness instead of the makespan. In general, PSO needs an initial set of particles to be initiated. Knowing that the performance of an algorithm is affected by the quality of its initial solution, two problem-specific particles based on the EDD rule and MDA are produced. These two particles are considered as a part of the set of initial swarms that is generated randomly. Computational experiments demonstrated that starting with the suggested initial set of particles results in better solutions than the randomly generated set. Generally, at a significance level of 0.05 , the suggested initial swarms (that contain the particles obtained using the EDD rule and MDA) performed better than the regular initial swarms (that were generated completely at random). Hence, we insert these two problem-specific particles into the initial set of particles, which is generated in Step 1.2 of the IGPSO algorithm. Moreover, GPSO has a set of input parameters. The main input parameters are the swarm size (the number of initially generated particles) and the weights $\left(w_{1}, w_{2}\right.$, and $\left.w_{3}\right)$. The other inputs are the mutation operator and stopping condition. The swarm size and the weights are taken from [30]. Because we are solving a different problem, we fine-tuned the parameters around the values taken from [30]. Three values are considered: the same value and a combination of smaller and larger values. The considered values and the selected values (based on the computational experiments) are presented in Table 1.

Table 1. Parameters for IGPSO and HGPSO

\begin{tabular}{ccc}
\hline Parameters & $\begin{array}{c}\text { Considered } \\
\text { Values }\end{array}$ & Selected Values \\
\hline Swarm Size & 30,50 , and 70 & 70 \\
\hline $\mathrm{w}_{1}$ & $0.1,0.2$, and 0.3 & 0.2 \\
\hline $\mathrm{w}_{2}$ & $0.35,0.4$, and 0.45 & 0.4 \\
\hline $\mathrm{w}_{3}$ & $0.35,0.4$, and 0.45 & 0.4 \\
\hline Mutation & - & $\begin{array}{c}\text { Insertion and swap } \\
\text { (combined) }\end{array}$ \\
\hline
\end{tabular}

The used mutation operator was set as a combination of the insertion and swap operators, and the stopping condition was set such that the computation times (in seconds) in all the algorithms were the same (or zero total tardiness was found). The test data were randomly generated as illustrated in Section 4. The IGPSO steps are summarized as follows:

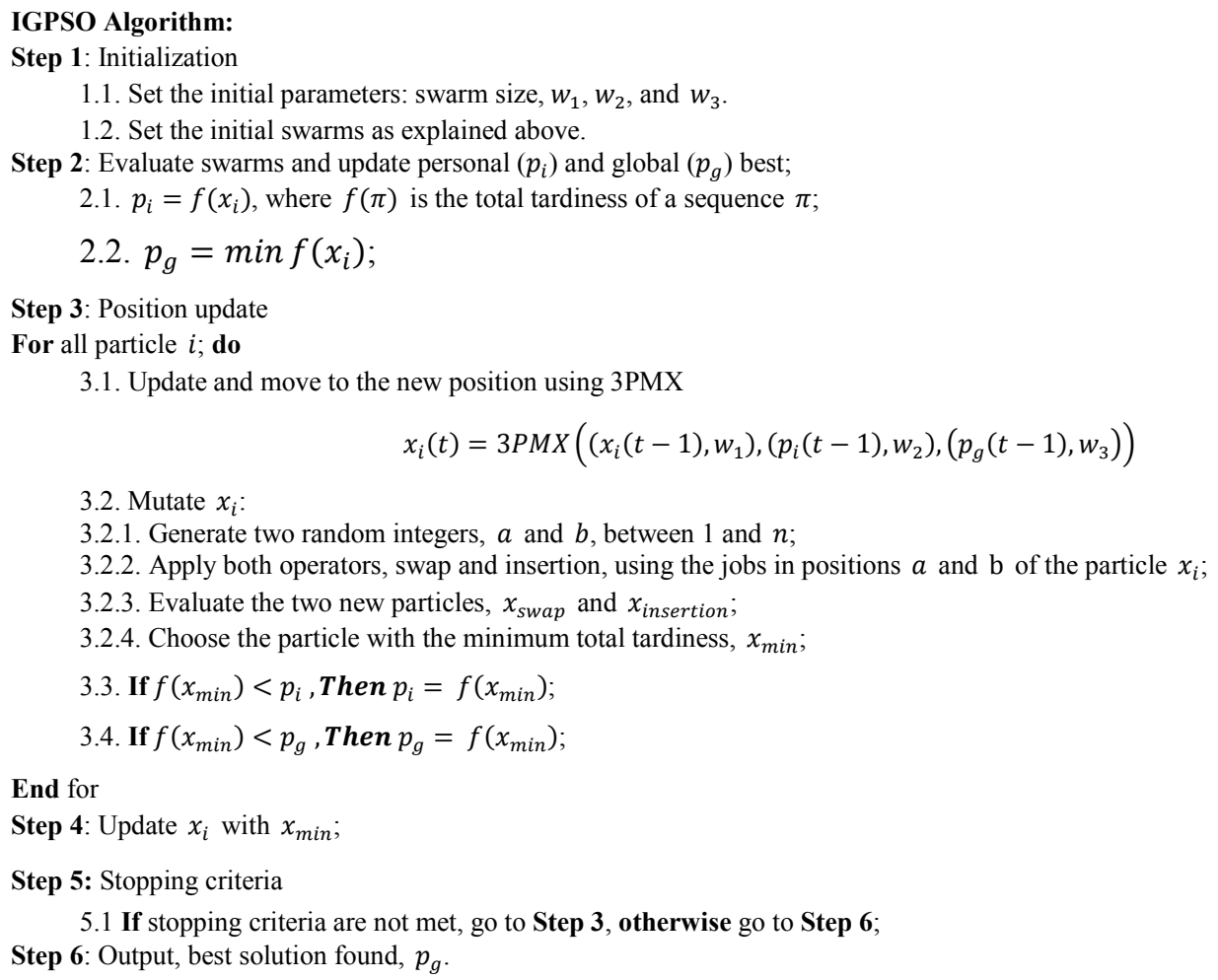


The HGPSO algorithm has the same inputs as the IGPSO algorithm, apart from some other inputs related to the SA algorithm. HGPSO differs from IGPSO in that HGPSO optimizes its initial swarms before advancing to the updating step. The optimization process of the initial swarms was conducted by simply inserting each swarm (or particle) as an initial solution to the SA algorithm. The employed SA algorithm is an adapted SA algorithm that is developed by [18] for solving the $F\left(m_{1}, m_{2}\right)|n w t| C_{\max }$. The only modification to the adapted SA was the decrement in the number of iterations (per temperature decrease) and the overall replications needed during the search process. If this decrement were not conducted, the developed HGPSO would be a time-consuming algorithm. Based on some computational experiments and the values reported by [18], the parameters set for the SA algorithm are presented in Table 2.

Table 2. Parameters Setting for the SA

\begin{tabular}{ccc}
\hline \multirow{2}{*}{ Parameters } & \multicolumn{2}{c}{ Considered Values } \\
\cline { 2 - 3 } & Small-scale & Large-scale \\
\hline Initial temperature $\left(T_{0}\right)$ & 200 & 400 \\
\hline Final temperature $\left(T_{f}\right)$ & 0.001 & 0.0001 \\
\hline Number of iterations & 200 & 500 \\
\hline$\alpha$ & 0.90 & 0.95 \\
\hline Stop condition & Reaching $\left(T_{f}\right),(50 \%)$ of the CPU time given to the HGPSO, or zero total tardiness \\
\hline
\end{tabular}

The steps of HGPSO are presented as follows:

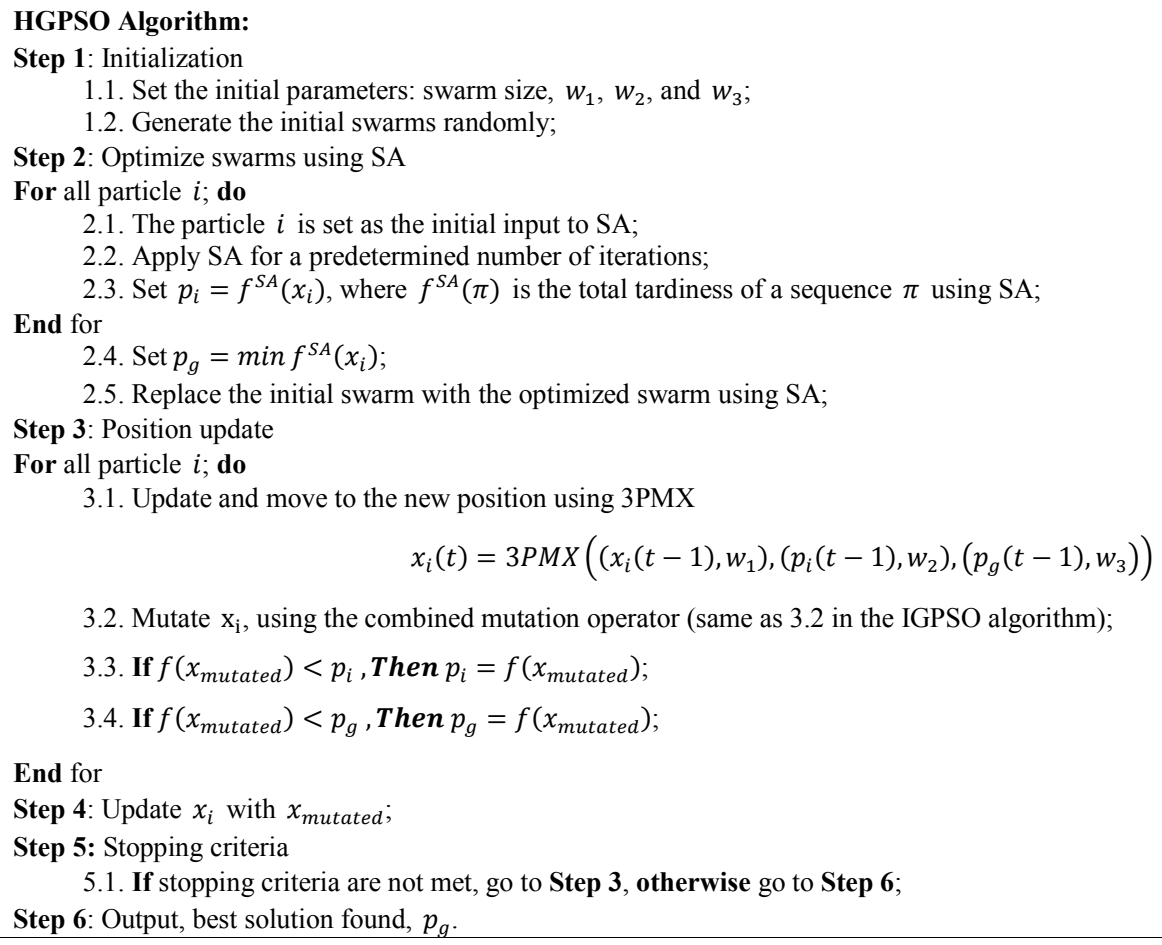

2.5. Replace the initial swarm with the optimized swarm using SA;

Step 3: Position update

For all particle $i$; do

3.1. Update and move to the new position using 3PMX

$$
x_{i}(t)=3 \operatorname{PMX}\left(\left(x_{i}(t-1), w_{1}\right),\left(p_{i}(t-1), w_{2}\right),\left(p_{g}(t-1), w_{3}\right)\right)
$$

3.2. Mutate $x_{i}$, using the combined mutation operator (same as 3.2 in the IGPSO algorithm);

$$
\begin{aligned}
& \text { 3.3. If } f\left(x_{\text {mutated }}\right)<p_{i}, \text { Then } p_{i}=f\left(x_{\text {mutated }}\right) \text {; } \\
& \text { 3.4. If } f\left(x_{\text {mutated }}\right)<p_{g}, \text { Then } p_{g}=f\left(x_{\text {mutated }}\right) \text {; }
\end{aligned}
$$

End for

Step 4: Update $x_{i}$ with $x_{\text {mutated }}$;

Step 5: Stopping criteria

5.1. If stopping criteria are not met, go to Step 3, otherwise go to Step 6;

Step 6: Output, best solution found, $p_{g}$.

As in all previously described algorithms, the stopping condition for the HGPSO was set to have the same CPU time in all the algorithms. 


\section{Results and discussion}

In this section, the computations and evaluations of the five designed algorithms are addressed. The algorithms were coded with MATLAB and run on a PC with an Intel ${ }^{\circledR}$ Core $^{\mathrm{TM}}$ i7-4930K CPU processor at $3.40 \mathrm{GHz}$, and $32.0 \mathrm{~GB}$ of RAM.

In order to evaluate the two newly introduced algorithms (IGPSO and HGPSO), a set of test problems was generated to evaluate the effectiveness of the IGPSO and HGPSO algorithms with the other adopted algorithms (ST-PSO, SMR-PSO, and MSRM-PSO), under different shop-floor conditions. Based on previous studies (e.g., [16]), the test problems were divided into two groups, which are smallscale and large-scale problems. Accordingly, the test problems design parameters took several values for each problem scale. We determined eight levels for the number of jobs for each problem scale. We also determined three levels of machine combinations for the number of identical parallel machines at each stage. The considered combinations are based on the assumption of whether the number of machines at each stage are balanced (equal). This assumption has been used in computational experiments in several previous studies (e.g., [9]). The processing times for jobs at both stages were generated randomly using uniform distributions of $U(4,40)$ and $U(60,300)$ for the small- and large-scale problems, respectively. [18] recommended this distribution and many authors have used it in their studies. The due dates were generated using a uniform distribution based on the formula reported in the work of [14]:

$$
d_{j}=p_{1 j}+p_{2 j}+\operatorname{ceil}\left(\frac{U(0,1) \times \sum_{j=1}^{n}\left(p_{1 j}+p_{2 j}\right)}{m_{1}+m_{2}}\right) \quad j=1,2, \ldots,
$$

where $\operatorname{ceil}(X)$ returns the smallest integer value greater than or equal to $X$, and $U(0,1)$ is a random number with standard uniform distribution. Table 3 summarizes the values of the considered variables for generating the test problems and conducting the experimental study.

As mentioned in Section 3, the performance of the designed algorithms was compared to the performance of the benchmark algorithms. $f_{\text {ins }}^{A_{i}}$ stands for the total tardiness value obtained by an algorithm $A_{i}$ for instance ins, and $f_{\text {ins }}^{\text {Best }}$ stands for the best-found value for instance ins. The best-found value is calculated as the minimum of the five algorithms.

$$
f_{\text {ins }}^{\text {Best }}=\min _{i} f_{\text {ins }}^{A_{i}}, i=1, \ldots, 5
$$

Then, a relative error ( $\mathrm{RE}$ ) can be used to assess the performance of the designed algorithms. RE is defined as follows:

$$
R E=\left|1-\frac{f_{\text {ins }}^{A_{i}}}{f_{\text {ins }}^{\text {Best }}}\right|
$$

In order to eliminate the effect of random variations, the simulation runs were repeated 10 times for each combination of variables. Extensive simulations were conducted based on the above-reported variables. The results of the test problems (with a total of $2 \times 3 \times 8 \times 5 \times$ $10=2400$ observations) were analyzed to find the best PSO variant to solve the considered problem. The CPU times for the five algorithms (ST-PSO, SMR-PSO, MSRMPSO, IGPSO, and HGPSO) were set to be the same, and thus it has not been included in the comparisons. The results of the conducted computations are presented in Tables 4 and 5, and all the results are illustrated in Figures 2-9. The figures show the performance of the five

\begin{tabular}{|c|c|c|c|}
\hline \# & Variables & Number of levels & The levels \\
\hline 1 & PS (Problem scale) & 2 & $\begin{array}{l}S: \text { Small-scale; } \\
L: \text { Large-scale; }\end{array}$ \\
\hline 2 & $m$ (Number of machines) & 3 & $\begin{array}{c}S:\left\{\begin{array}{c}m_{1}=3, m_{2}=3, \text { balanced } m_{1}=m_{2} \\
m_{1}=3, m_{2}=4, \text { unbalanced } m_{1}<m_{2} \\
m_{1}=4, m_{2}=3, \text { unbalanced } m_{1}>m_{2}\end{array}\right. \\
L:\left\{\begin{array}{c}m_{1}=8, m_{2}=8, \text { balanced } m_{1}=m_{2} \\
m_{1}=8, m_{2}=10, \text { unbalanced } m_{1}<m_{2} \\
m_{1}=10, m_{2}=8, \text { unbalanced } m_{1}>m_{2}\end{array}\right.\end{array}$ \\
\hline 3 & $n$ (Number of jobs) & 8 & $\begin{array}{l}S: n=\{10,20,30,40,50,60,70,80\} \\
L: n=\{100,300,500,700,900,1000,1500,2000\}\end{array}$ \\
\hline 4 & $P T$ (Processing times) & 1 & $\begin{array}{l}S: U(4,40) \\
L: U(60,300)\end{array}$ \\
\hline 5 & $A_{i}$ (Algorithms) & 5 & $\begin{array}{l}A_{1}: \text { ST-PSO; } \\
A_{2}: \text { SMR-PSO; } \\
A_{3}: \text { MSRM-PSO; } \\
A_{4}: \text { IGPSO; } \\
A_{5}: \text { HGPSO; }\end{array}$ \\
\hline
\end{tabular}
algorithms in terms of the average relative error.

Table 3. Included variables in test problems design. 
Table 4. Relative error results for the small-scale problems

\begin{tabular}{|c|c|c|c|c|c|c|c|}
\hline \multirow{2}{*}{ Jobs } & \multicolumn{2}{|c|}{ Machines } & \multicolumn{5}{|c|}{ Average relative error } \\
\hline & $m_{1}$ & $m_{2}$ & ST-PSO & SMR-PSO & MSPM-PSO & IGPSO & HGPSO \\
\hline \multirow{3}{*}{10} & 3 & 3 & 1.130 & 1.609 & 1.000 & 1.000 & 1.000 \\
\hline & 3 & 4 & 1.068 & 1.091 & 1.000 & 1.000 & 1.000 \\
\hline & 4 & 3 & 3.000 & 1.000 & 1.000 & 1.000 & 1.000 \\
\hline \multirow{3}{*}{20} & 3 & 3 & 2.372 & 2.718 & 3.478 & 1.084 & 1.000 \\
\hline & 3 & 4 & 1.315 & 1.668 & 1.969 & 1.023 & 1.000 \\
\hline & 4 & 3 & 1.832 & 2.085 & 2.323 & 1.015 & 1.000 \\
\hline \multirow{3}{*}{30} & 3 & 3 & 4.720 & 5.528 & 7.664 & 1.280 & 1.000 \\
\hline & 3 & 4 & 1.499 & 1.699 & 2.163 & 1.041 & 1.000 \\
\hline & 4 & 3 & 2.310 & 2.675 & 3.445 & 1.195 & 1.000 \\
\hline \multirow{3}{*}{40} & 3 & 3 & 2.439 & 2.691 & 3.473 & 1.128 & 1.000 \\
\hline & 3 & 4 & 1.461 & 1.663 & 1.993 & 1.075 & 1.000 \\
\hline & 4 & 3 & 2.203 & 2.505 & 3.039 & 1.101 & 1.000 \\
\hline \multirow{3}{*}{50} & 3 & 3 & 2.916 & 3.591 & 4.578 & 1.205 & 1.000 \\
\hline & 3 & 4 & 1.526 & 1.760 & 2.092 & 1.081 & 1.000 \\
\hline & 4 & 3 & 2.494 & 2.677 & 3.406 & 1.122 & 1.000 \\
\hline \multirow{3}{*}{60} & 3 & 3 & 2.470 & 2.862 & 3.472 & 1.331 & 1.000 \\
\hline & 3 & 4 & 1.559 & 1.731 & 2.084 & 1.109 & 1.000 \\
\hline & 4 & 3 & 1.955 & 2.126 & 2.697 & 1.114 & 1.000 \\
\hline \multirow{3}{*}{70} & 3 & 3 & 2.585 & 3.089 & 3.627 & 1.358 & 1.000 \\
\hline & 3 & 4 & 1.600 & 1.853 & 2.176 & 1.133 & 1.000 \\
\hline & 4 & 3 & 2.351 & 2.583 & 3.195 & 1.259 & 1.000 \\
\hline \multirow{3}{*}{80} & 3 & 3 & 2.401 & 2.699 & 3.343 & 1.388 & 1.000 \\
\hline & 3 & 4 & 1.591 & 1.796 & 2.126 & 1.158 & 1.000 \\
\hline & 4 & 3 & 1.880 & 2.093 & 2.483 & 1.212 & 1.000 \\
\hline
\end{tabular}

Table 5. Relative error results for the large-scale problems

\begin{tabular}{|c|c|c|c|c|c|c|c|}
\hline \multirow{2}{*}{ Jobs } & \multicolumn{2}{|c|}{ Machines } & \multicolumn{5}{|c|}{ Average relative error } \\
\hline & $m_{1}$ & $m_{2}$ & ST-PSO & SMR-PSO & MSPM-PSO & IGPSO & HGPSO \\
\hline \multirow{3}{*}{100} & 8 & 8 & 2.930 & 3.074 & 3.639 & 1.315 & 1.000 \\
\hline & 8 & 10 & 1.671 & 1.837 & 2.093 & 1.084 & 1.000 \\
\hline & 10 & 8 & 2.566 & 2.642 & 3.116 & 1.107 & 1.000 \\
\hline \multirow{3}{*}{300} & 8 & 8 & 1.470 & 1.606 & 1.713 & 1.103 & 1.000 \\
\hline & 8 & 10 & 1.271 & 1.377 & 1.445 & 1.027 & 1.000 \\
\hline & 10 & 8 & 1.385 & 1.472 & 1.546 & 1.058 & 1.000 \\
\hline \multirow{3}{*}{500} & 8 & 8 & 1.311 & 1.381 & 1.407 & 1.109 & 1.000 \\
\hline & 8 & 10 & 1.259 & 1.326 & 1.346 & 1.096 & 1.000 \\
\hline & 10 & 8 & 1.273 & 1.336 & 1.352 & 1.076 & 1.000 \\
\hline \multirow{3}{*}{700} & 8 & 8 & 1.267 & 1.301 & 1.312 & 1.104 & 1.000 \\
\hline & 8 & 10 & 1.227 & 1.266 & 1.270 & 1.088 & 1.000 \\
\hline & 10 & 8 & 1.238 & 1.273 & 1.277 & 1.094 & 1.000 \\
\hline \multirow{3}{*}{900} & 8 & 8 & 1.244 & 1.257 & 1.261 & 1.095 & 1.000 \\
\hline & 8 & 10 & 1.209 & 1.221 & 1.226 & 1.090 & 1.000 \\
\hline & 10 & 8 & 1.203 & 1.226 & 1.230 & 1.095 & 1.000 \\
\hline \multirow{3}{*}{1000} & 8 & 8 & 1.237 & 1.247 & 1.248 & 1.094 & 1.000 \\
\hline & 8 & 10 & 1.183 & 1.196 & 1.198 & 1.074 & 1.000 \\
\hline & 10 & 8 & 1.186 & 1.198 & 1.199 & 1.079 & 1.000 \\
\hline \multirow{3}{*}{1500} & 8 & 8 & 1.165 & 1.167 & 1.167 & 1.071 & 1.000 \\
\hline & 8 & 10 & 1.153 & 1.153 & 1.154 & 1.070 & 1.000 \\
\hline & 10 & 8 & 1.146 & 1.147 & 1.147 & 1.062 & 1.000 \\
\hline \multirow{3}{*}{2000} & 8 & 8 & 1.127 & 1.128 & 1.128 & 1.055 & 1.000 \\
\hline & 8 & 10 & 1.109 & 1.111 & 1.111 & 1.054 & 1.000 \\
\hline & 10 & 8 & 1.109 & 1.110 & 1.110 & 1.051 & 1.000 \\
\hline
\end{tabular}


For the small-scale problems, Figure 2 shows the overall performance of the five algorithms, which clearly demonstrate that both the IGPSO and HGPSO algorithms performed better than the ST-PSO, SMR-PSO, and MSRM-PSO algorithms. Moreover, considering only the three adopted algorithms, ST-PSO was the best.

Figures 3 and 4 illustrate the performance of the five designed algorithms with different numbers of jobs and machines (for the small-scale problems). It can be concluded that the ST-PSO, SMR-PSO, and MSRM-PSO algorithms performed considerably worse than the IGPSO and HGPSO algorithms. Similarly, the ST-PSO algorithm performed better than the SMR-PSO and MSRM-PSO algorithms. Moreover, with the increase in the number of jobs from 10 to 80 the performance of HGPSO and IGPSO were considerably better than the benchmark algorithms (see Figure 3). The same conclusion can be drawn by analyzing the results with a different number of machines at each stage (see Figure 4).

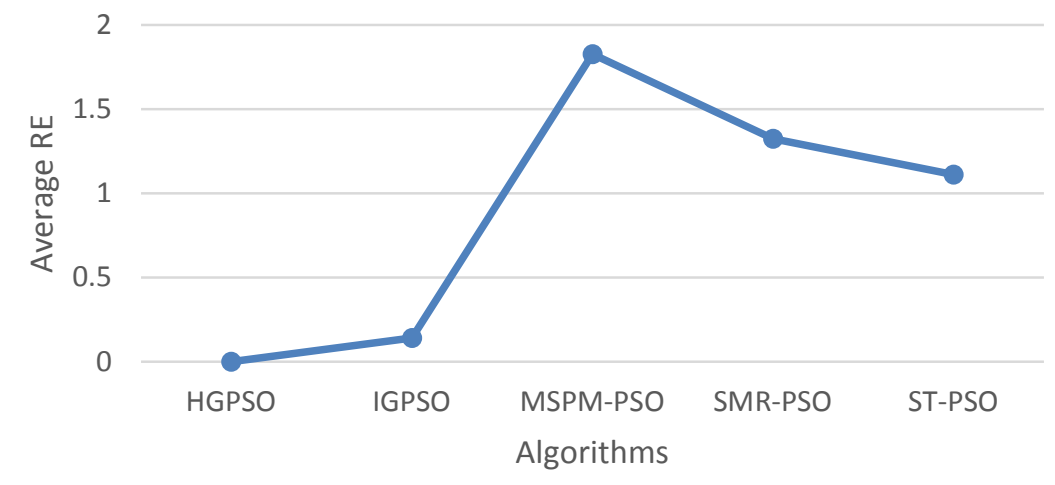

Figure 2. Average RE versus algorithms for the small-scale problems

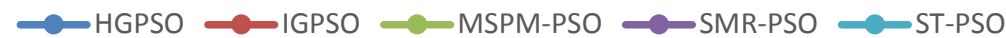

4

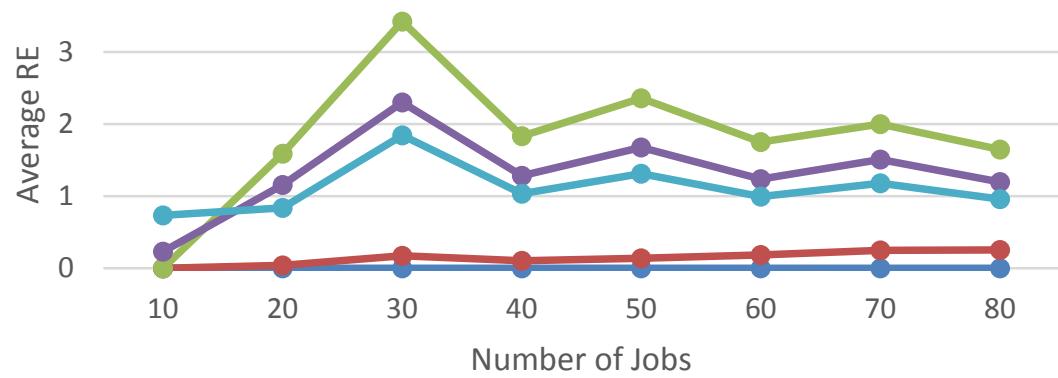

Figure 3. Average RE versus the number of jobs for all algorithms for the small-scale problems

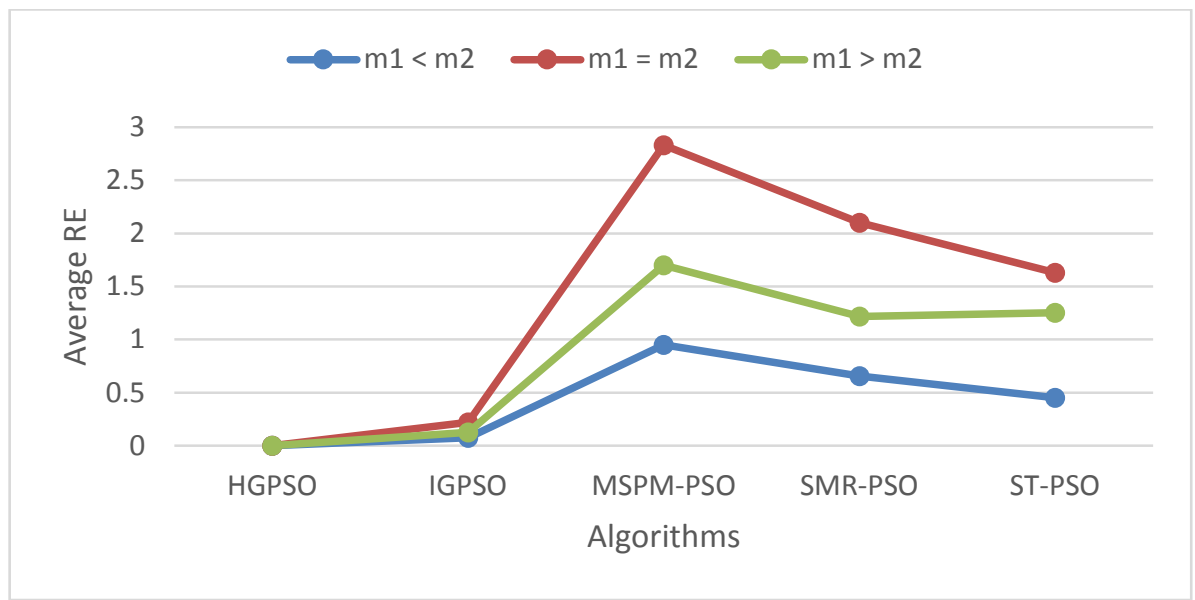

Figure 4. Average RE Versus Algorithms for Different Numbers of Machines Each Stage for the Small-scale Problems 
Figure 4 also shows that when the number of machines in both stages were balanced (equal), the instances where difficult to solve compared to other instances when the number of machines in both stages were unbalanced (not equal). As shown in Figures 2-4, the IGPSO and HGPSO algorithms seemed to exhibit comparable performance; however, the HGPSO algorithm showed superior performance to IGPSO, as indicated by Figure 5 .

Figure 5 was obtained by excluding the performance of the ST-PSO, SMR-PSO, and MSRM-PSO algorithms from Figure 3 to deliver a more accurate comparison. Figure 5 obviously demonstrates that the average relative error versus the number of jobs with HGPSO was smaller than that with IGPSO for the small-scale problems.

For the large-scale problems, the same conclusions (as in the small-scale) can be drawn, as shown in Figures 6-9.

The overall relative errors when solving the small-scale problems using the ST-PSO, SMR-PSO, MSRM-PSO, IGPSO, and HGPSO algorithms were 1.111, 1.325, 1.826, 0.142 , and 0.0 , respectively. When solving the large-scale problems, the overall relative errors using the ST-PSO, SMR-PSO, MSRM-PSO, IGPSO, and HGPSO algorithms were $0.372,0.419,0.487,0.090$, and 0.0 , respectively. In general, the overall relative errors using the five abovementioned algorithms for solving the considered problem were $0.742,0.872,1.157,0.116$, and 0.0 , respectively. This indicates that, compared to the best benchmark algorithm (ST-PSO), IGPSO was $84 \%$ smaller in terms of the overall relative error. This demonstrates the higher performance of the proposed IGPSO algorithm. Moreover, the overall relative error with HGPSO was zero, which shows the superiority of the proposed HGPSO algorithm compared to all the other algorithms.

In order to confirm the obtained results, a full factorial design for relative error versus jobs, machines, and algorithms was analyzed. The analysis of variance (ANOVA) results (for both small- and large-scale problems) assessed the effects of jobs, machines, and algorithms on the relative error. All the p-values were less than 0.05 , which indicated a significant effect of the three factors (number of jobs, number of machines at each stage, and used algorithm) on the average relative error. The five algorithms were compared with the Dunnett simultaneous test at $95 \%$ confidence intervals in order to confirm our conclusions about their average performance. Figure 10 shows the results of the Dunnett simultaneous test. The results confirmed our conclusions that IGPSO and HGPSO outperform the other three algorithms. Moreover, it was found that the difference between the average performance of IGPSO and HGPSO is not significant, which means that IGPSO and HGPSO mostly showed similar performance.

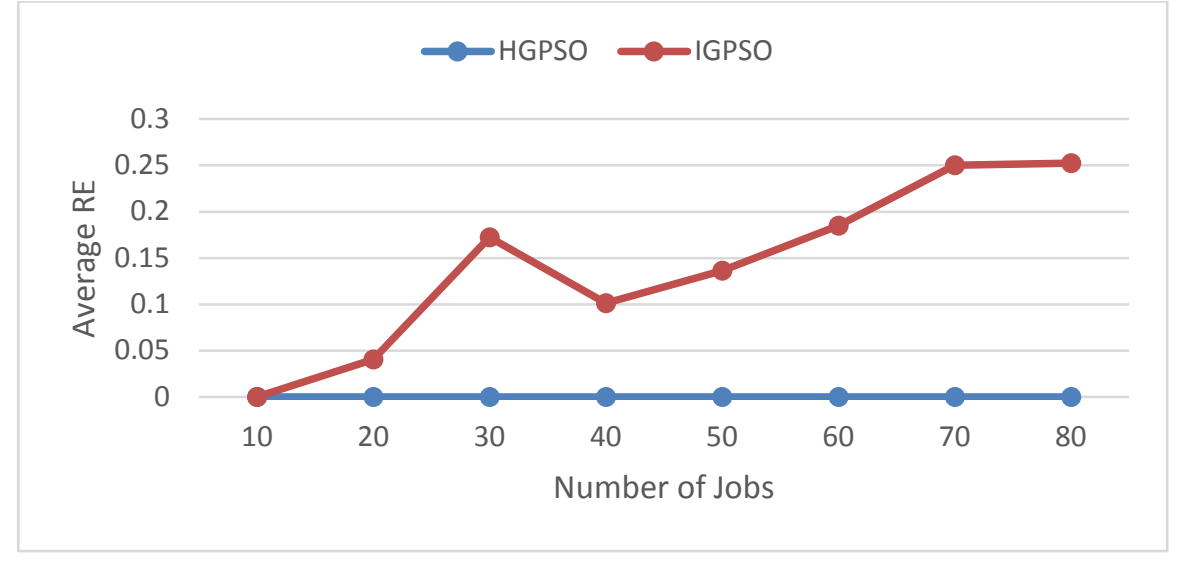

Figure 5. Average RE versus number of jobs for IGPSO and HGPSO for the small-scale problems

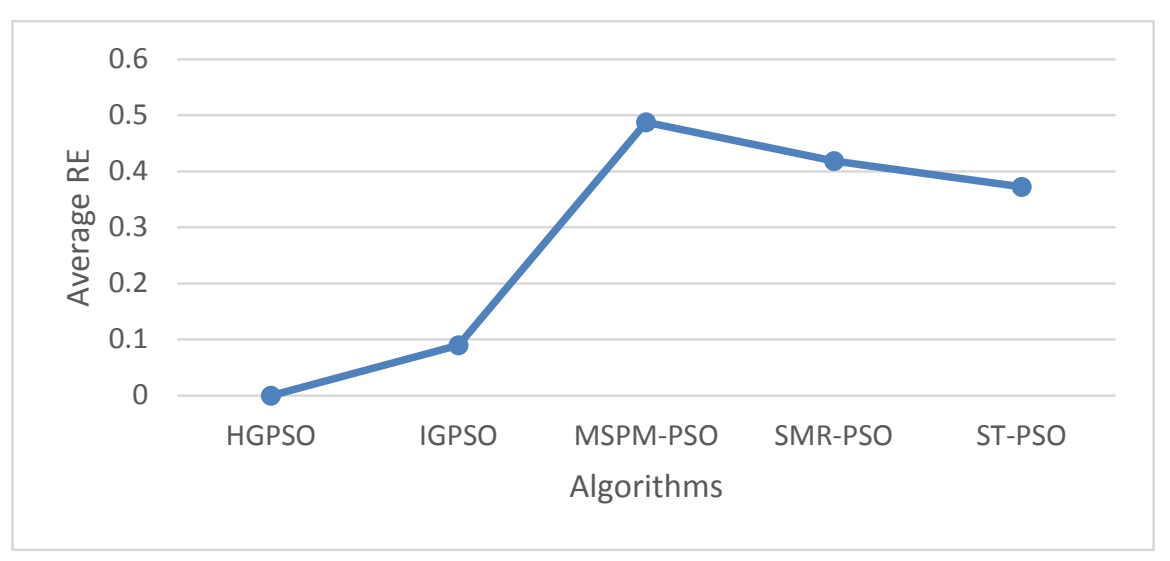

Figure 6. Average RE versus algorithms for the large-scale problems 


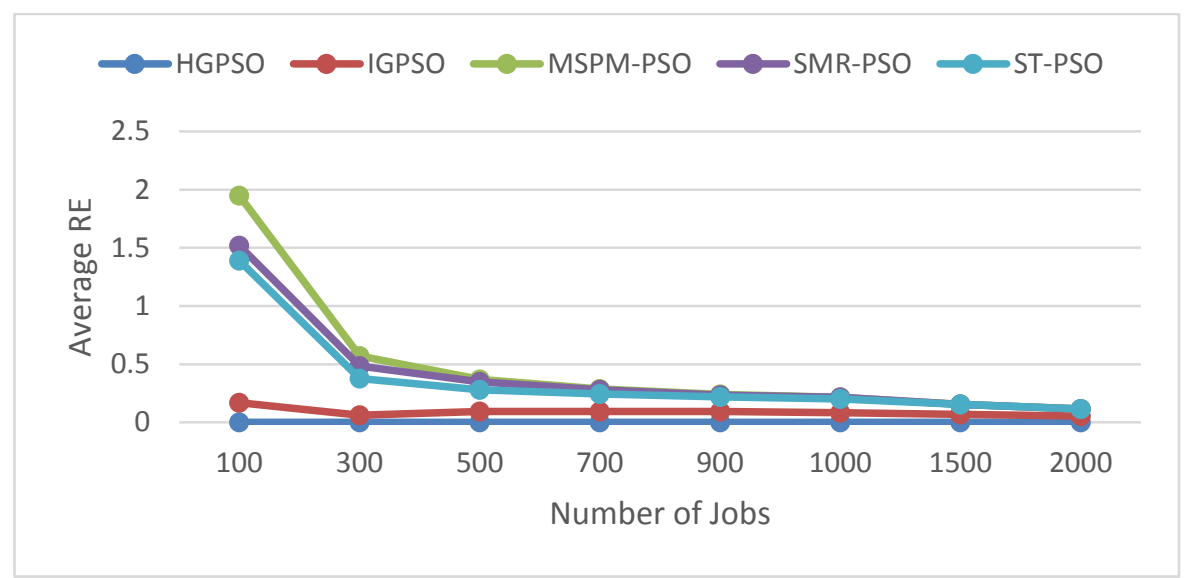

Figure 7. Average RE versus number of jobs for all algorithms for the large-scale problems

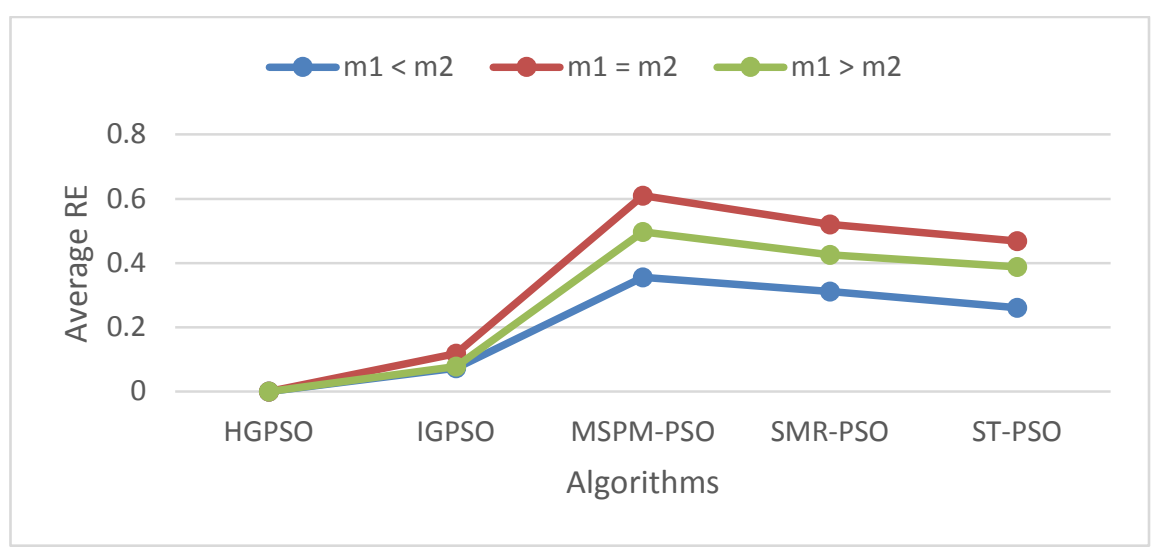

Figure 8. Average RE versus algorithms for the number of machines each stage for the large-scale problems

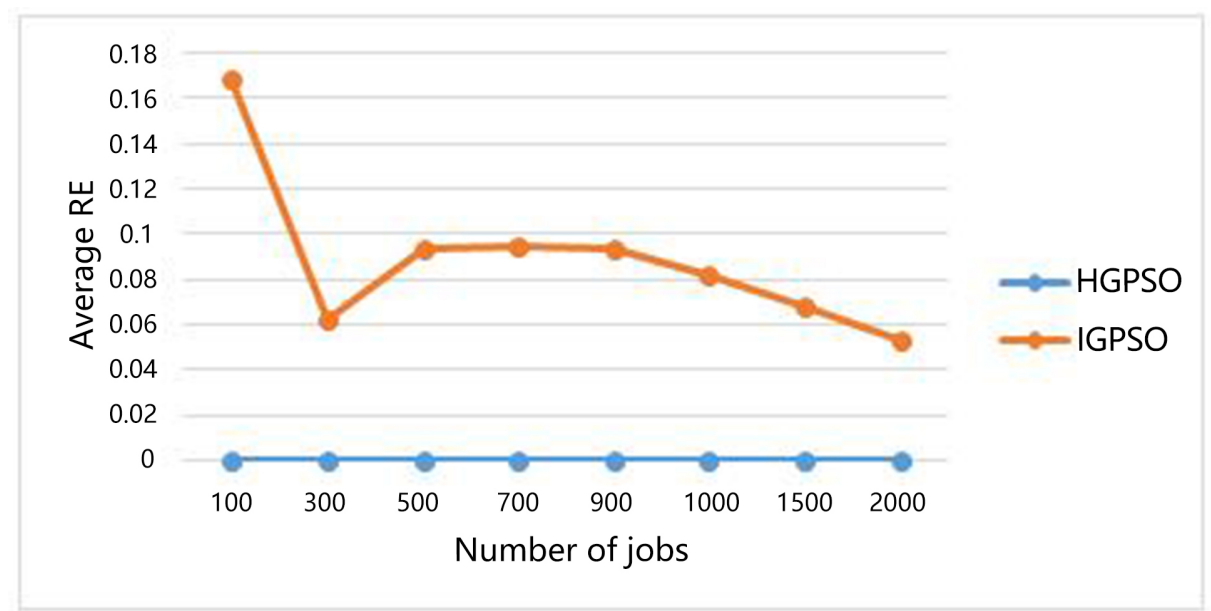




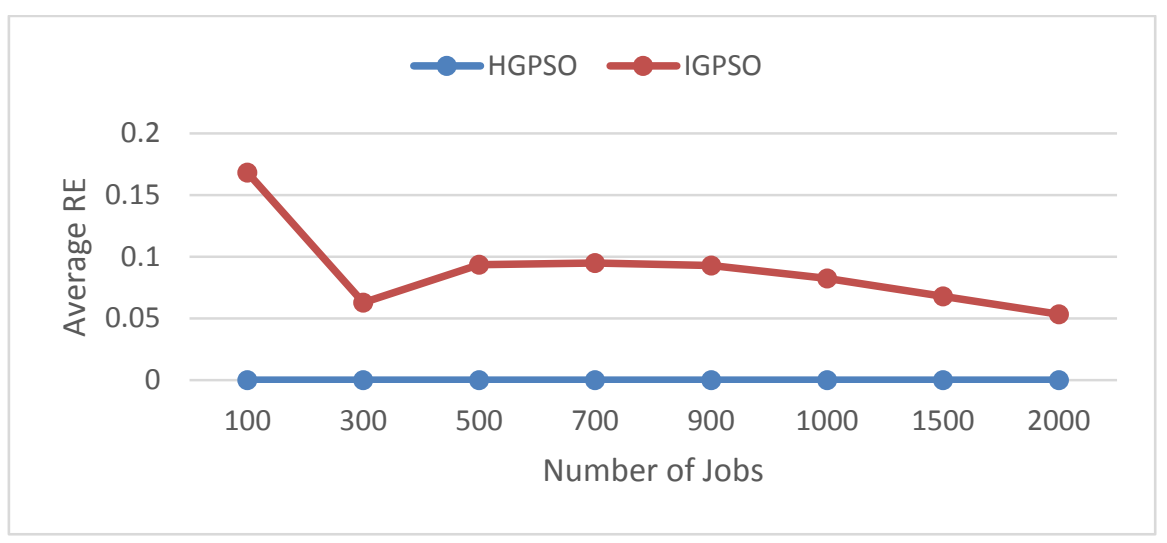

Figure 9. Average RE versus number of jobs for IGPSO and HGPSO for the large-scale problems.

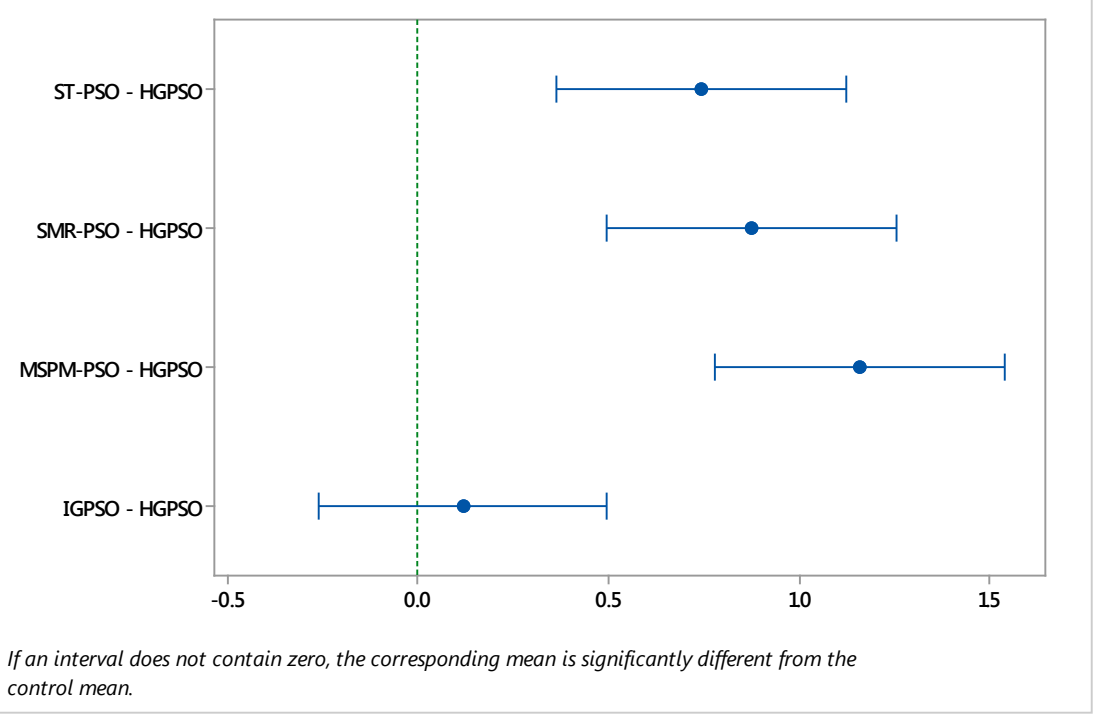

Figure 10. Dunnett Simultaneous with $95 \%$ Confidence Intervals.

Therefore, the proposed IGPSO and HGPSO are recommended for minimizing the total tardiness in the hybrid flowshop scheduling problems with no-wait, and we highly recommend HGPSO because this algorithm obtained zero relative error for all the solved instances.

\section{Conclusions}

In this article, we studied the two-stage NWHFSP with the objective of minimizing the total tardiness. The considered problem was proved to be NP-hard in the strong sense. Three different variants of the PSO were adopted and two new variants were proposed. The ST-PSO, SMR-PSO, and MSRM-PSO were proved to have good performance according to prior studies for solving the considered problem with different objective functions. The IGPSO and HGPSO are the two newly introduced algorithms that we proposed for solving the problem under investigation. Moreover, we mathematically formulated the problem and proposed two problem-specific initial solutions. Extensive computational experiments have been carried out in order to evaluate the performance of the five algorithms. Generally, it has been found that the initially generated swarms have a significant effect on the quality of the bestfound solutions (in all the algorithms). However, the effect was considerably small in some instances.

The results showed that the overall relative errors when solving the considered problem using the ST-PSO, SMRPSO, MSRM-PSO, IGPSO, and HGPSO algorithms were $0.742,0.872,1.157,0.116$, and 0.0 , respectively. Therefore, the IGPSO and HGPSO algorithms clearly outperformed the ST-PSO, SMR-PSO, and MSRM-PSO algorithms. Moreover, considering only the adopted algorithms, STPSO performed better than the SMR-PSO and MSRM-PSO algorithms. In particular, the IGPSO algorithm reduced the relative error obtained by ST-PSO, which was found to have the smallest relative error among the three adopted algorithms, by $84 \%$, and HGPSO obtained zero relative error in all instances. Therefore, the use of IGPSO and HGPSO is recommended.

In this study, we assumed that setup times are included 
with the processing times and machines are available all the time, which is unrealistic for some manufacturing systems. Therefore, scope exists to extend the study to include setup times, machine breakdown, or both. Moreover, the study has been restricted to the two-stage case. Therefore, another possible extension could include cases with more than two stages. A further possible extension could be to utilize the two newly proposed algorithms in solving the considered problem with multiple objectives.

\section{Conflict of Interest}

The authors declare that there is no conflict of interest regarding the publication of this paper.

\section{Data Availability}

The data used to support the findings of this study are included in the article.

\section{Funding Statement}

This work was supported by the research center of the college of engineering at King Saud University.

\section{Acknowledgements}

The authors thank the Deanship of Scientific Research and RSSU at King Saud University for their technical support. The authors would like to thank all personnel involved in this work.

\section{REFERENCES}

[1] M. Salvador, "A solution of a special class of flow shop problems,” Process Symp. Theory Sched. Its Appl., pp. 8391, 1973.

[2] S. Guirchoun, P. Martineau, and J. Billaut, "Total completion time minimization in a computer system with a server and two parallel processors," Comput. Oper. Res., vol. 32, pp. 599-611, 2005.

[3] J. Song and J. Tang, "Scheduling model and discrete particle swarm optimization algorithm for roller annealing," 2008 Asia Simul. Conf. - 7th Int. Conf. Syst. Simul. Sci. Comput., pp. 770-775, 2008.

[4] R. H. Huang, C. L. Yang, and Y. C. Huang, "No-wait two-stage multiprocessor flow shop scheduling with unit setup,” Int. J. Adv. Manuf. Technol., vol. 44, no. 9-10, pp. 921-927, 2009.
[5] Z. Liu, J. Xie, J. Li, and J. Dong, “A heuristic for two-stage no-wait hybrid flowshop scheduling with a single machine in either stage,” Tsinghua Sci. Technol., vol. 8, no. 1, pp. 43-48, 2003.

[6] J. Chang, W. Yan, and H. Shao, "Two-Stage No-Wait Hybrid Flowshop With Separated Setup and Removal Times," in Proceeding of the 2004 American Control Conference, 2004, pp. 1412-1416.

[7] S. Wang and M. Liu, "A genetic algorithm for two-stage no-wait hybrid flow shop scheduling problem," Comput. Oper. Res., vol. 40, no. 4, pp. 1064-1075, 2013.

[8] S. Wang, M. Liu, and C. Chu, "A branch-and-bound algorithm for two-stage no-wait hybrid flow-shop scheduling,” Int. J. Prod. Res., vol. 53, no. 4, pp. 11431167, 2015.

[9] N. Moradi, R. Shafaei, M. Rabiee, and M. Mazinani, "Minimization of maximum tardiness in a no-wait two stage flexible flow shop,” Int. J. Artif. Intell., vol. 8, no. 12 S, pp. 166-181, 2012.

[10] N. Moradinasab, R. Shafaei, M. Rabiee, and P. Ramezani, "No-wait two stage hybrid flow shop scheduling with genetic and adaptive imperialist competitive algorithms," $J$. Exp. Theor. Artif. Intell., no. May 2015, pp. 1-19, 2012.

[11] M. Rabiee, R. Sadeghi Rad, M. Mazinani, and R. Shafaei, "An intelligent hybrid meta-heuristic for solving a case of no-wait two-stage flexible flow shop scheduling problem with unrelated parallel machines," Int. J. Adv. Manuf. Technol., vol. 71, no. 5-8, pp. 1229-1245, 2014.

[12] A. Adressi, S. T. Hassanpour, and V. Azizi, "Solving group scheduling problem in no-wait flexible flowshop with random machine breakdown," Decis. Sci. Lett., vol. 5, no. 1, pp. 157-168, 2016.

[13] S. Carpov, J. Carlier, D. Nace, and R. Sirdey, “Two-stage hybrid flow shop with precedence constraints and parallel machines at second stage,” Comput. Oper. Res., vol. 39, no. 3, pp. 736-745, 2012.

[14] F. Jolai, H. Asefi, M. Rabiee, and P. Ramezani, "Bi-objective simulated annealing approaches for no-wait two-stage flexible flow shop scheduling problem," Sci. Iran., vol. 20, no. 3, pp. 861-872, 2013.

[15] C. Sriskandarajah, "Performance of scheduling algorithms for no-wait flowshops with parallel machines," Eur. J. Oper. Res., vol. 70, no. 3, pp. 365-378, 1993.

[16] J. Xie, W. Xing, Z. Liu, and J. Dong, "Minimum deviation algorithm for two-stage no-wait flowshops with parallel machines," Comput. Math. with Appl., vol. 47, no. 12, pp. 1857-1863, 2004.

[17] Z. Wang, W. Xing, and F. Bai, "No-wait flexible flowshop scheduling with no-idle machines,” Oper. Res. Lett., vol. 33, no. 6, pp. 609-614, 2005.

[18] M. Rabiee, P. Ramezani, and R. Shafaei, “An efficient simulated annealing algorithm for a No-Wait Two Stage Flexible Flow Shop Scheduling Problem,” vol. 1, no. 4, pp. 13-28, 2011.

[19] R. Shafaei, M. Rabiee, and M. Mirzaeyan, “An adaptive neuro fuzzy inference system for makespan estimation in multiprocessor no-wait two stage flow shop," Int. J. 
Comput. Integr. Manuf., vol. 24, no. 10, pp. 888-899, 2011.

[20] R. Shafaei, N. Moradinasab, and M. Rabiee, "Efficient meta heuristic algorithms to minimize mean flow time in no-wait two stage flow shops with parallel and identical machines," Int. J. Manag. Sci. Eng. Manag., vol. 6, no. 6, pp. 421-430, 2011.

[21] M. A. Ghaleb, U. S. Suryahatmaja, and I. M. Alharkan, "Metaheuristics for two-stage no-wait flexible flow shop scheduling problem," in IEOM 2015 - 5th International Conference on Industrial Engineering and Operations Management, Proceeding, 2015.

[22] A. Allahverdi, H. Aydilek, and A. Aydilek, “Two-stage assembly scheduling problem for minimizing total tardiness with setup times,” Appl. Math. Model., vol. 40, no. 17-18, pp. 7796-7815, 2016.

[23] M. Gourgand, N. Grangeon, and S. Norre, "Meta-heuristics for the deterministic hybrid flow shop problem," J. Eur. Des Syst. Autom., vol. 34, no. 9, pp. 1107-1136, 2000.

[24] J. A. Hoogeveen, J. K. Lenstra, and B. Veltman, "Preemptive scheduling in a two-stage multiprocessor flow shop is NP-hard,” Eur. J. Oper. Res., vol. 89, no. 1, pp. 172175, 1996.

[25] R. Eberhart and J. Kennedy, “A new optimizer using particle swarm theory," MHS'95. Proc. Sixth Int. Symp. Micro Mach. Hum. Sci., pp. 39-43, 1995.

[26] K. P. Wang, L. Huang, C. G. Zhou, and W. Pang, "Particle swarm optimization for traveling salesman problem," in Machine Learning and Cybernetics, 2003, pp. 1583-1585.

[27] Y. W. Zhao, B. Wu, W. L. Wang, Y. L. Ma, W. A. Wang, and H. Sun, "Particle swarm optimization for vehicle routing problem with time windows," in Advances in Materials Manufacturing Science and Technology, 2004, pp. 801-805.

[28] Y. Jia, J. Qu, and L. Wang, “A Novel Particle Swarm Optimization Algorithm for Permutation Flow-Shop Scheduling Problem,” vol. 8944, no. 301, pp. 15-25, 2016.

[29] T. El-Ghazali, Metaheuristics from design to implementation. New Jersey: John Wiley \& Sons, Inc., 2009.

[30] A. Moraglio, C. Di Chio, J. Togelius, and R. Poli, “Geometric Particle Swarm Optimization,” J. Artif. Evol. Appl., vol. 2008, pp. 1-14, 2008. 